J. Clin. Chem. Clin. Biochem.

Vol. 20,1982 , pp. $287-289$

\title{
The Relationship of HDL-Apolipoprotein A-I and HDL-Cholesterol to Risk Factors of Coronary Heart Disease: Initial Results of the Prospective Epidemiological Study in Company Employees in Westfalia
}

By G. Assmann, H. Funke and H. Schriewer

Zentrallaboratorium der Medizinischen Einrichtungen der Westfälischen Wilhelms-Universität Münster

und

Institut für Arterioskleroseforschung an der Universität Münster

(Received October 8/December 10, 1981)

Summary: In a prospective epidemiologic study in company employees in Westfalia aimed at improving early diagnosis of coronary heart disease in 3069 men and 1387 women, concentràtions of HDL-apolipoprotein A-I and HDL-cholesterol were measured and the results were correlated with several risk factors of coronary heart disease. A negative correlation between hypertriglyceridaemia and HDL-cholesterol $(\mathrm{r}=-0.250 ;-0.27 \% ; \mathrm{p}<0.001)$ and between relative body weight and HDL-cholesterol $(r=-0.21 \delta ;-0.20 \% ; p<0.001)$ could be shown but not between hypertriglyceridaemia and HDL-apolipoprotein A-I and between relative body weight and HDL-apolipoprotein A-I. In cigarette smokers HDL-apolipoprotein A-I as well as HDL-cholesterol were found to be lower than in non-smokers. On the other hand HDL-apolipoprotein A-I but not HDL-cholesterol was positively correlated with systolic and diastolic blood pressure.

Die Beziehung von HDL-Apolipoprotein A-I und HDL-Cholesterin zu Risikofaktoren der koronaren Herzkrankheit: Erste.Ergebnisse der prospektiven epidemiologischen Studie bei Betriebsangehörigen im Raum Westfalen

Zusammenfassung: In einer prospektiven epidemiologischen Studie bei Betriebsangehörigen im Raum Westfalen zur Verbesserung der Frühdiagnostik der koronaren Herzkrankheit wurden die Konzentrationen von HDL-Apolipoprotein A-I und HDL-Cholesterin bei 3069 Männern und 1387 Frauen untersucht und die Ergebnisse mit den verschiedenen Risikofaktoren der koronaren Herzkrankheit korreliert. Nur für HDL-Cholesterin, aber nicht für HDLApolipopiōtein A-I wurde eine negative Korrelation zur Triglyceridkonzentration $\left(r=-0,25 \delta^{*} ;-0,27 q ; p<0,001\right)$. und zum relativen Körpergewicht $(r=-0,21 \delta ;-0,20 \% ; p<0,001)$ beobachtet. Bei Zigarettenrauchern waren sowohl die Konzentrationen von HDL-Apolipoprotein A-I als âuch von HDL-Cholesterin niedriger als bei Nichtrauchern. Auf der anderen Seite war HDL-Apolipopprotein A-I, nicht jedoch HiDL-Cholesterin, positiv mit dem systolischen und diastolischen Blutdruck korreliert.

\section{Introduction}

In epidemiological $(1-5)$ and clinical studies $(6-7)$ the determination of HDL-cholesterol in addition to the analysis of total cholesterol and triglycerides has been shown to improve the detection of the risk of coronary heart disease. The possible relationship of other HDL constituents (e.g. apolipoproteins A-I and A-II, phosphatidylcholine, sphingomyelin) to the risk of coronary heart disease is not yet known. Though several clinical studies have indicated decreased levels of apolipoprotein A-I in subjects with coronary heart disease $(7,8,11)$, there is still a lack of prospective epidemiological data to demonstrate the predictive power of HDL-apolipoproteins with respect to coronary heart disease.

The present paper reports initial results of our epidemiological studies (12) with regard to the correlation of HDL-apolipoprotein A-I with several risk factors of coronary heart disease.

\section{Subjects and Methods}

3069 male and 1387 female company employees from four different companies in the Westfalia region were studied ("Prospective epidemiologic study in company employees in 
Westfalia"). In a special bus, blood was collected in the morning after a $12 \mathrm{~h}$ tast and was allowed to clot for $30 \mathrm{~min}$ at room temperature. After removing cells by centrifugation at $\mathbf{3 0 0 0}$ $\min ^{-1}$. the serum was stored at $4^{\circ} \mathrm{C}$ and was transported to our laboratory within 3 days maximum. Experiments were performed within 25 hours after receiving the samples. Serum levels of cholesterol, triglycerides, glucose and uric acid were determined with the SMAC Autoanalyzer (Technicon GmbH., Bad Vilbel, GFR) as described elsewhere (12).

For determination of HDL-cholesterol, apolipoprotein B-containing lipoproteins were precipitated using the phosphotungstate $/ \mathrm{MgCl}_{2}$ precipitation method (13). LDL-cholesterol was calculated from total cholesterol, and HDL-cholesterol and triglycerides according to the formula of Friedewald (14). HDL-apolipoprotein A-I was measured by rate nephelometry. Details have been described elsewhere (13).

\section{Results}

The means and ranges for HDL-apolipoprotein A-I and HDL-cholesterol for males and females in our prospective epidemiological study are shown in table 1. Females exhibited obviously higher levels of HDL-apolipoprotein A-I and HDL-cholesterol than males. In both sexes levels of HDL-apolipoprotein A-I and HDLcholesterol were normally distributed.

The results of the correlation analysis of HDL-cholesterol, HDL-apolipoprotein A-I, age and several risk factors of coronary heart disease are shown in table 2 .

In males and females HDL-apolipoprotein A-I levels were positively correlated with age, while a positive correlation between HDL-cholesterol and age was found in females but not in males. Furthermore, in both sexes there were obviously significant positive correlations between HDLapolipoprotein A-I and HDL-cholesterol (males $r=0.66$, females $\mathrm{r}=0.68)$, HDL-apolipoprotein $\mathrm{A}-\mathrm{I}$ and serum cholesterol (males $r=0.21$, females $r=0.27$ ), HDL-apolipoprotein A-I and uric acid (males $r=0.12$, females $\mathrm{r}=0.08$ ) and HDL-apolipoprotein A-I and systolic blood pressure (males $r=0.07$, females $r=0.11$ ). A negative correlation was calculated between HDL-apolipoprotein

Tab. 1. HDL-apolipoprotein A-I and HDL-cholesterol concentration in company employees in Westfalia.

\begin{tabular}{|c|c|c|c|c|}
\hline & \multicolumn{2}{|c|}{$\begin{array}{l}\text { HDL-apolipo- } \\
\text { protein A-I } \\
(\mathrm{g} / \mathrm{l})\end{array}$} & \multicolumn{2}{|c|}{$\begin{array}{l}\text { HDL-cholesterol } \\
(\mathrm{g} / \mathrm{l})\end{array}$} \\
\hline & $\begin{array}{l}\text { men } \\
n=3032\end{array}$ & $\begin{array}{l}\text { women } \\
n=1387\end{array}$ & $\begin{array}{l}\text { men } \\
n=3069\end{array}$ & $\begin{array}{l}\text { women } \\
n=1380\end{array}$ \\
\hline $\begin{array}{l}\text { Mean } \\
\text { Standard deviation } \\
\text { Median } \\
\text { Minimum } \\
\text { Maximum } \\
5 \% \text { percentile } \\
25 \% \text { percentile } \\
75 \% \text { percentile } \\
95 \% \text { percentile }\end{array}$ & $\begin{array}{l}1.363 \\
0.231 \\
1.348 \\
0.61 \\
2.44 \\
1.02 \\
1.21 \\
1.50 \\
1.76\end{array}$ & $\begin{array}{l}1.469 \\
0.252 \\
1.457 \\
0.74 \\
2.66 \\
1.07 \\
1.30 \\
1.63 \\
1.88\end{array}$ & $\begin{array}{l}0.4384 \\
0.1179 \\
0.4196 \\
0.16 \\
1.47 \\
0.27 \\
0.36 \\
0.50 \\
0.65\end{array}$ & $\begin{array}{l}0.5326 \\
0.1382 \\
0.5185 \\
0.21 \\
1.15 \\
0.33 \\
0.43 \\
0.61 \\
0.77\end{array}$ \\
\hline
\end{tabular}

Tab. 2. Coefficients of correlation between HDL-cholesterol and risk factors and between HDL-apolipoprotein A-I and risk factors (prospective epidemiological study in company employees in Westfalia).

\begin{tabular}{|c|c|c|c|c|}
\hline & \multicolumn{2}{|c|}{ Males, $n=3032$} & \multicolumn{2}{|c|}{ Females, $n=1380$} \\
\hline & $\begin{array}{l}\text { HDL- } \\
\text { choles- } \\
\text { tẹrol }\end{array}$ & $\begin{array}{l}\text { HDL- ; } \\
\text { apo- } \\
\text { lipo- } \\
\text { protein } \\
\text { A-I }\end{array}$ & $\begin{array}{l}\text { HDL- } \\
\text { choles- } \\
\text { terol }\end{array}$ & $\begin{array}{l}\text { HDL- } \\
\text { apo- } \\
\text { lipo- } \\
\text { protein } \\
\text { A-I }\end{array}$ \\
\hline Age & 0.03 & $0.12^{3}$ & $0.14^{3}$ & $0.18^{3}$ \\
\hline HDL-cholesterol & & $0.66^{3}$ & & $0.68^{3}$ \\
\hline $\begin{array}{l}\text { HDL-apolipoprotein- } \\
\text { A-I }\end{array}$ & $0.66^{3}$ & & $0.68^{3}$ & \\
\hline LDL-cholesterol & $-0.05^{2}$ & 0.03 & -0.02 & 0.04 \\
\hline Cholesterol . & $0.09^{3}$ & $0.21^{3}$ & $0.23^{3}$ & $0.27^{3}$ \\
\hline Triglycerides & $=0.25^{3}$ & 0.01 & $-0.27^{3}$ & 0.03 \\
\hline Glucose & $-0.05^{1}$ & $0.06^{2}$ & $-0: 08^{2}$ & 0.03 \\
\hline Uric acid & -0.02 & $0.12^{3}$ & $-0.09^{2}$ & $0.08^{2}$ \\
\hline $\begin{array}{l}\text { Systolic blood } \\
\text { pressure }\end{array}$ & 0.03 & $0.07^{3}$ & 0.00 & $0.11^{3}$ \\
\hline $\begin{array}{l}\text { Diastolic blood } \\
\text { pressure }\end{array}$ & -0.00 & $0.04^{1}$ & -0.00 & $0 ! 08^{1}$ \\
\hline $\begin{array}{l}\text { Relative body weight } \\
\text { (Broca-index) }\end{array}$ & $-0.21^{3}$ & $0: 00$ & $-0.20^{3}$ & $=0.00$ \\
\hline Cigarette smoking & $=0.05^{1}$ & $-0.09^{3}$ & $-0.11^{3}$ & $=0.12^{3}$ \\
\hline
\end{tabular}

1) $\mathrm{p}<0.05$

2) $\mathrm{p}<0.01$

3) $\mathrm{p}<0.001$

A- $\dot{I}$ and cigarette smoking (males $r=-0.09$, females $\mathrm{r}=-0.12$ ).

In contrast to HDL-äpolipoprotein A-I, HDL-cholesterol was not positively correlâted with uric acid or systolic and diastolic blood pressure. In both sexes HDLcholesterol showed a close negative correlation with triglycerides (males $r=-0.25$, females $r=-0.27$ ), bôdy weight (males $r \equiv-0.21$, females $r=-0.20$ ) and cigarette smoking (males $r=-0.05$, females $r=-0.11$ ). The most prominent result was the lack of a negative correlation between HDL-apolipoprotein A-I and triglycerides or HDL-apolipoprotein A-I ànd bödy weight, respectively, in males and females.

\section{Discussion}

High density lipoproteins comprise a heterogenous mixture of macromolecules which differ. with règard to particle size, chemical composition and physicochemical properties. It is impossible to calculate $\mathrm{HDL}$ mass from HDL-cholesterol because the content of the cholesterol moiety in HDL is ranged between 10-20\% of HDL mass. In hypertriglyceridaemic sera the cholesterol content of HDL particles is lowered while the triglyceride content of these particles is enhanced (15). 
Natrium/KaliumBestimmung sofort

ofne Flamme onhe Probenvobiereitung

Der Natrium/Kalium.Analyzer von Jookoo ist sofort einsatzbe reit. Onne probenvorbereitung werden Natrium und Kalium aus Blut, "Serum, Plasma oder Urin präzise bestimmt speziell entwickelte ionenselektive Elektro. den mit langer Lebensdauer und hoher Empfindlichkeit, automa tische Funktionskontrolle und Eichung durch Mikroprozessor Probenwechsler für 30 Proben.

Mehr erfahren sie aus unseren Informationsunterlagen.

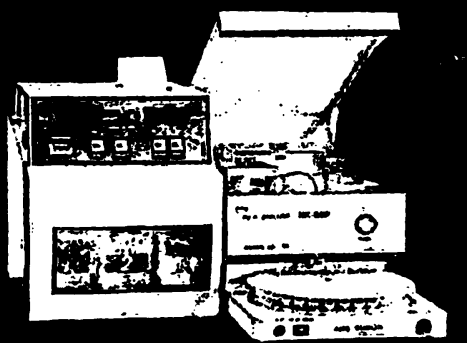

\section{Fettstoffwechsel- Diagnostik}

neบ] (1)

Triglyceside)

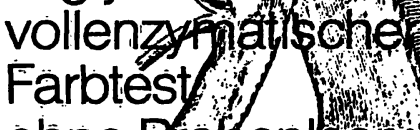
ohne probenteen
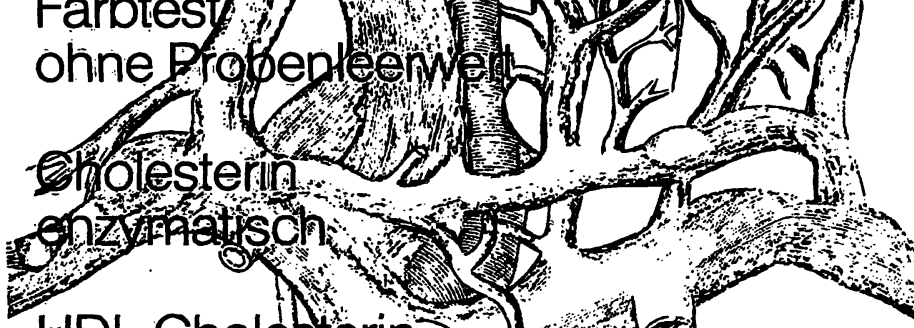

HDL-Cholesterin -

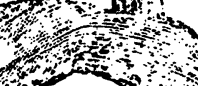
$1 \mathrm{M}$
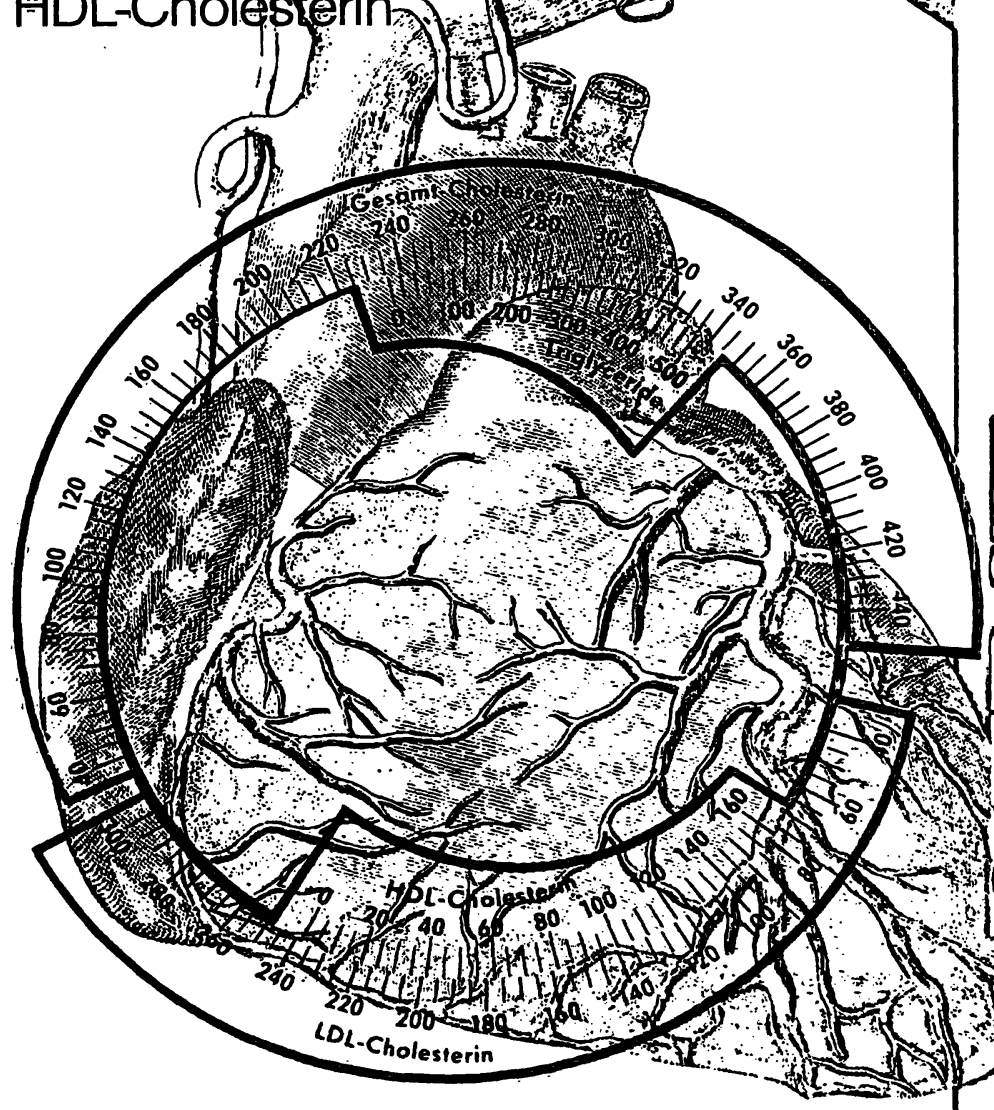

Aussagen über das Risiko arteriosklerotischer Gefäßerkrankungen sind durch die Bestimmung des Gesamtcholesterins und der Triglyceride im Serum möglich; darum sind diese beiden Bestimmungen als Basisprogramm der Lipiddiagnostik anzusehen. Die zusätzliche Bestimmung des HDLCholesterins erlaubt weitere fundierte diagnostische Aussàgen. 


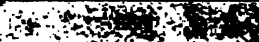

Fast

Hemoglobins do Reflect Blood Glucose

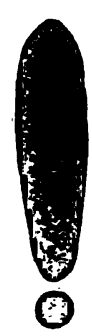

Current research reports demonstrate a good correlation between the chromatographically fast hemoglobins (HbA 1a, 1b and 1c) and time-averaged sugar levelts.

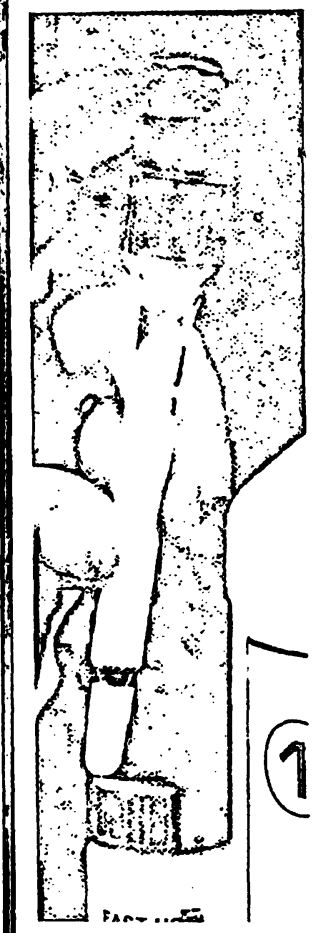

FAST

HEMOGLOBIN TEST SYSTEM

Now European-wide available with " $\mathrm{HbA}_{1}$ Aldimin Eliminator": as hemolysation reagent Since we have been the first, our experience is not to beat

Therefore:

FAST HEMOGLOBIN TEST SYSTEM the method of choice for glycohemoglobin determination from

"patents pending

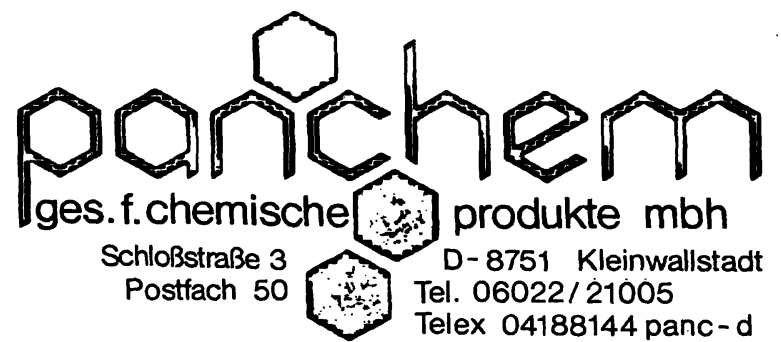

or nearest distributor: Benelux: Biolab. Limal. Denmärk: Struers, Copenhagen. Finland: Medix. Helsinki. France: Laboratoire Eurobio, Paris. Haly: Soc. Mascia Brunelli, Milano Sweden: Bio-Bol. Handen. Switzerland: Bühlmann Laboratories, Basel. United Kingdòm: Advanced Laboratories Techniques. Tunbridge Wells Universa Scientific, London. Austria: Medizin-Labortechnik Politakis. Innsbruck. Greece: Vass. Messimeris, Athens.

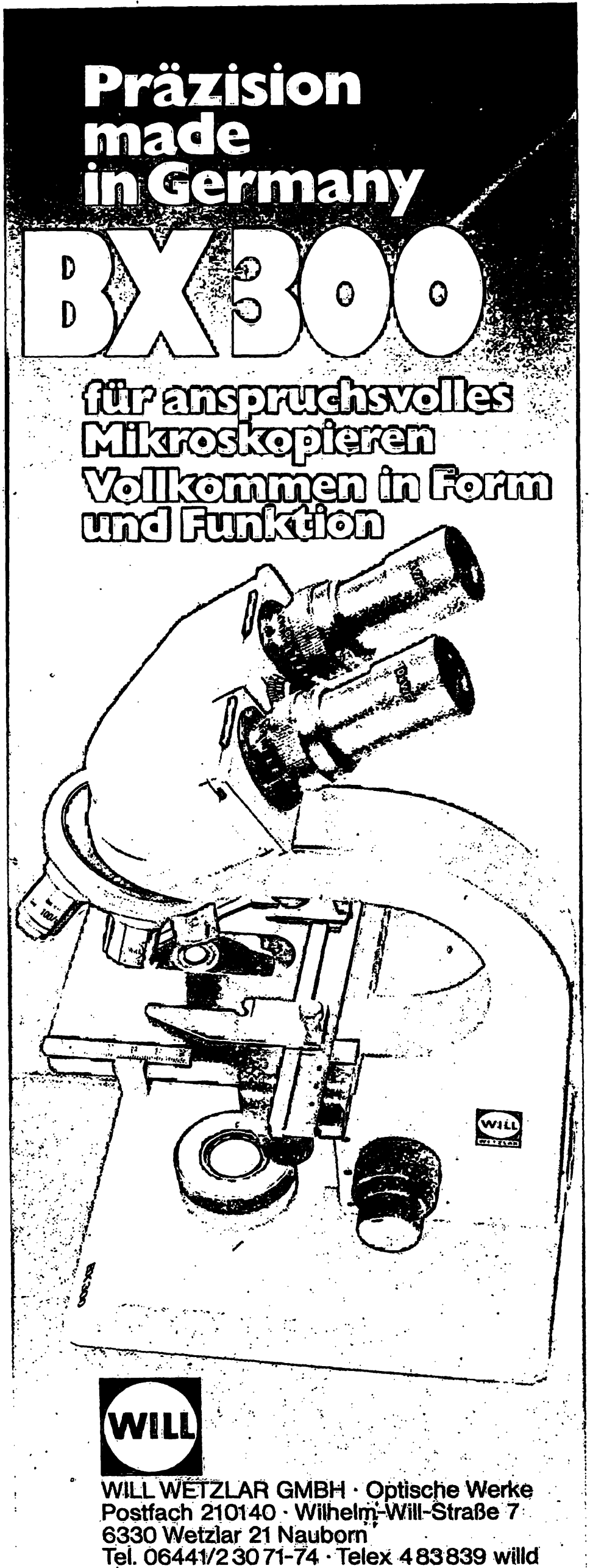

Anfragen und Prospektanforderungen richten Sie bitte an Abteilung 66 
In light of the heterogeneity of HDL the well-known association between HDL-cholesterol and coronary heart disease does not necessarily reflect a similar association between HDL mass and coronary heart disease or between HDL-apolipoproteins and coronary heart disease. In contrast to HDL-cholesterol the predictive power of other HDL constituents, especially HDL-apolipoproteins, has not been established in prospective studies. According to studies by Avogaro et al. on survivors of myocardial infarction (10), or Bradby et al. on patients with peripheral vascular disease (8), HDL-apolipoprotein A-I may be a better discriminator between atherosclerotic subjects and controls than HDL-cholesterol.

In other clinical reports, however $(9,11)$, the superiority of HDL-apolipoprotein A-I as compared to HDLcholesterol could not be shown. The preliminary results of our prospective epidemiological study show that HDL- cholesterol and HDL-apolipoprotein A-I may exhibit a different relationship to coronary risk.

The negative correlation between HDL-cholesterol and hypertriglyceridaemia and between HDL-cholesterol and obesity could not be demonstrated for HDL-apolipoprotein A-I. In cigarette smokers HDL-cholesterol as well as HDL-apolipoprotein A-I were found to be lower than in non-smokers.

On the other hand HDL-apolipoprotein A-I but not HDL-cholesterol was positively correlated with systolic and diastolic blood pressure. The possible implications of these observations remain to be determined.

\section{Acknowledgement}

Supported by the Bundesministerium für Forschung und Technologie, Bonn.

\section{References}

1. Miller, G. J. \& Miller, N. E. (1975) Lancet $I, 16-19$.

2. Rhoads, G. G., Gulbrandsen, C. L. \& Kagan, A. (1976) New Engl. J. Med. 294, 293-298.

3. Berg, K., Bфrresen, A. \& Dahlén, G. (1976) Lancet $I$, $499-502$.

4. Castelli, W. P., Doyle, J. T., Gordon, T., Hames, C. G., Hjortlañd, M. C., Hulley, S. B., Kagañ, A. \& Zukel, W. J. (1977) Circulation 55, 767-772.

5. Gordon, T., Castelli, W. P. Hjortland, M. C., Kannel, W. B. \& Dawber, T. R. (1977) Ann. Int. Med. 87, 393-397.

6. Barboriak, J. J., Anderson, A. J., Rimm, A. A. \& King, J. F. (1979) Metabólism 28, 735-738.

7. Kladetzky, R. G., Assmann, G., Walgenbach, S., Tauchert, P. \& Helb, H.-D. (1980) Artery 7, 191-205.

8. Bradbỳ, G. V. H., Valente, A. J. \& Wạltón, K. W. (1978) Lancet $I, 1271-1274$.

9. Albers, J. J., Cheung, M. C. \& Hazzard, W. R. (1978) Metabolism 27, 479-485.

10. Avorago, P., Bon, G. B., Cazzalato, G. \& Quinci, G. B. (1979) Lancet $I, 901-903$.

11. Ishikawa, T., Fidge, N., Thelle, D. S., Forde, O. H. \& Miller, N. E. (1978) Eur. J. Clin. Invest. 8, 179-182.

12. Assmanni, G., Oberwittler, W., Schule, H., Schriewer, H., Funke, H., Epping, P. H. \& Hauss, W. H. (1980) Internist $21,446-459$.

13. Assmann, G., Schriewer, H. \& Funke, H. (1981) J. Clin. Chem. Clin. Biochem. 19, 273-278.

14. Friedewald, W. T., Levy, R. I. \& Fredrickson, D. S. (1972) Clin. Chem. 18, 499-509.

15. Herbert, P. N. \& Henderson, L. O. (1979) Lancet $I$, $1368-1370$.
Profiessor Dr. G. Assmann

Zentrallaboratorium

der Medizinischen Einrichtungen der Westfälischen Wilhelms-Universität Domagkstraße 3

D-4400 Münster 
